\title{
Corrigendum: Postsynaptic current bursts instruct action potential firing at a graded synapse
}

\author{
Ping Liu, Bojun Chen \& Zhao-Wen Wang
}

Nature Communications 4:1911 doi: 10.1038/ncomms2925 (2013); Published 28 May 2013; Updated 14 May 2014

The legend to Fig. 5 of this Article contains a typographical error. Cholinergic PSCs in panel a were conducted using pipette solution I not III. The correct version of the legend appears below.

Figure 5 | Optogenetically evoked cholinergic and GABAergic PSC bursts depended on specific postsynaptic receptors. (a,b) Left: sample traces from worms raised in the presence of all-trans retinal showing the effects of (+)-TBC $(0.5 \mathrm{mM})$ and gabazine $(0.5 \mathrm{mM})$ on optogenetically evoked cholinergic and GABAergic PSC bursts, respectively. The horizontal blue lines represent blue light pulses while the " ${ }^{\prime \prime}$ sign indicates that the peak was cut off. Right: the persistent current of evoked cholinergic $(n=15)$ and GABAergic $(n=12)$ bursts were abolished by TBC and gabazine, respectively. (c) The persistent current of evoked cholinergic PSC bursts was greatly decreased in unc-29(e1072) but unchanged in acr-16(ok789) compared with wild type (WT). (d) The large initial transient of evoked cholinergic PSC bursts was greatly decreased in acr-16(ok789) but unchanged in unc-29(e1072)

compared with WT. In c,d, the sample size (n) was eight for every group. The WT group is the same as that shown in Fig. 4 . The asterisk $\left({ }^{\star}\right)$ indicates a statistically significant difference $(P<0.01)$ compared with either the control period in $\mathbf{a}$ or $\mathbf{b}$ (paired t-test) or the WT group in $c$ and $d$ (one-way analysis of variance followed by Bonferroni post hoc test). In $\mathbf{a}$ and $\mathbf{b}$, extracellular solution I and pipette solution I were used to record cholinergic PSCs whereas extracellular solution II and pipette solution II were used to record GABAergic PSCs. In $\mathbf{c}$ and $\mathbf{d}$, extracellular solution I and pipette solution I were used. 